\title{
Nonminimal gravitational coupling of scalar bound states
}

\author{
M. Reuter \\ Deutsches Elektronen-Synchrotron (DESY), Hamburg, Germany
}

(Received 22 November 1993)

\begin{abstract}
We use the large- $N$ approximation to investigate the nonminimal gravitational coupling $\xi R \phi^{2}$ of the scalar bound state in the $\mathrm{O}(N)$-symmetric vector model with a $\varphi^{4}$ interaction. If the elementary scalar field is minimally coupled to gravity, $\xi$ is found to assume values in the interval $[-1,0]$ only. For the conformally invariant coupling of the elementary boson, the bound state is always minimally coupled $(\xi=0)$. The induced $\xi$ term is also calculated for spontaneous and explicit symmetry breaking from $\mathrm{O}(N)$ to $\mathrm{O}(N-1)$ when the resulting Goldstone bosons can form bound states. The nonminimal gravitational coupling of scalars has important applications in inflationary cosmology.
\end{abstract}

PACS number(s): 98.80.Cq, 04.50. $+\mathrm{h}$

\section{INTRODUCTION}

It is well known that a scalar field in a curved spacetime, beyond its minimal coupling to gravity, also allows for a nonminimal coupling via an operator of dimension $4^{1}$ :

$$
S=\frac{1}{2} \int d^{4} x \sqrt{g} \phi\left[-D^{2}+\mu^{2}+\xi R(x)\right] \phi+S_{\mathrm{int}} .
$$

Here, $D_{\mu}$ is the covariant derivative containing the Christoffel symbols of the background metric $g_{\mu \nu}(x)$, and $R(x)$ is the corresponding Ricci scalar. Usually, the coefficient of the nonminimal term $\xi$ is treated as a free parameter on the same footing as masses and coupling constants. The only distinguished values for $\xi$ are $\xi=0$ corresponding to minimal coupling and $\xi=\frac{1}{6}$ which leads to a conformally invariant action if $\mu^{2}=0$. Recently it was pointed out [1] that if $\phi$ is a composite particle, the value of $\xi$ should be fixed by the underlying dynamics of the constituents. Specifically, it was shown that for the scalar composite boson of the Nambu-Jona-Lasinio model [2], the parameter $\xi$ can be calculated unambiguously. Remarkably, in a leading large- $N$ approximation one finds exactly the conformal value $\xi=\frac{1}{6}$. (Because of the scale-breaking effects due to the masses, this does not mean that the theory is conformally invariant.) A closely related question, the renormalization-group flow of $\xi$ in grand unified theories (GUT's), has also been studied [3-5].

The determination of $\xi$ in a given model is not only interesting in its own right but it also has interesting applications to various inflationary cosmologies. For instance, if $\xi$ is very large and negative $\left(\xi \approx-10^{4}\right)$, the Higgs field of grand unified theories could be used to drive inflation [6]. The extended inflation model of Ref. [7], on the other hand, favors much smaller values $(-0.01<\xi<0)$.

It is the aim of the present paper to study the induced $\xi$ term in two closely related models in which the formation of bound states can be treated analytically. The first one is the $\mathrm{O}(N)$-invariant vector model with a $\varphi^{4}$ interac-

\footnotetext{
${ }^{1}$ We use Euclidean notation throughout. Our conventions are $R_{\nu \alpha \beta}^{\mu}=\partial_{\alpha} \Gamma_{\nu \beta}^{\mu}-\cdots$ and $R=g^{\alpha \beta} R_{\alpha \mu \beta}^{\mu}$.
}

tion. The (bare) action reads

$S=\int d^{4} x \sqrt{g}\left\{\frac{1}{2} \varphi^{a}\left[-D^{2}+\mu_{0}^{2}+\hat{\xi} R\right] \varphi^{a}+\frac{\lambda_{0}}{4 ! N}\left(\varphi^{a} \varphi^{a}\right)^{2}\right\}$,

where $\varphi^{a}, a=1, \ldots, N$, is a vector under $\mathrm{O}(N)$. Using the large- $N$ expansion [8-12], it was shown (in flat space) that the inverse propagator of the composite operator $\varphi^{a} \varphi^{a}$ develops a zero at a certain (Euclidean) momentum $p^{2}=-m_{B}^{2}$ signaling the existence of an $\mathrm{O}(N)$-singlet, scalar bound state of mass $m_{B}$. Contrary to the Nambu-Jona-Lasinio model, the constituent particles are scalars themselves, so that there can be a $\hat{\xi} R$ term at the fundamental level already. In Sec. II we shall calculate the induced $\xi$ coupling of the composite particle for all values of $\hat{\xi}$ and the other renormalized parameters of the model. It turns out that $\xi$ depends on them only via the ratio $m / m_{B}$ where $m$ and $m_{B}$ are the masses of the elementary $\varphi^{a}$ boson and the bound state, respectively.

The renormalized form of the $\varphi^{4}$ theory, with the cutoff removed, should be considered a kind of toy model because most probably a nontrivial theory obtains only for a finite cutoff [11]. However, keeping the cutoff finite, one is left with a branch of solutions of the gap equation [10] for which the bound state is a tachyon. Nevertheless, the discussion in Sec. II provides a useful warmup for the slightly more complicated effective-field-theory model of Sec. III. It is obtained from (2), with $\mu_{0}^{2}<0$, by adding a term which breaks the $\mathrm{O}(N)$ symmetry to $\mathrm{O}(N-1)$ explicitly. Under certain conditions the Goldstone bosons related to this symmetry breaking form scalar bound states whose induced $\xi$ term we shall compute. Originally this model was inspired by the "surrogate Higgs boson" which exists in some technicolor models [13], and it was analyzed in detail by Chivukula and Golden [14].

\section{THE O(N)-SYMMETRIC VECTOR MODEL}

We analyze the model (2) generalizing the standard techniques developed for flat space [8-10]. Introducing an auxiliary field $\chi$ in order to linearize the quartic interaction term, the generating functional reads 


$$
\begin{aligned}
\exp \left\{\boldsymbol{W}\left[J^{a}\right]\right\} \equiv & \int D \varphi \exp \left\{-S[\varphi]+\int d^{4} x \sqrt{g} J^{a} \varphi^{a}\right\} \\
& =\int D \chi \int D \varphi^{a} \exp \left\{-\int d^{4} x \sqrt{g}\left(\frac{1}{2} \varphi^{a} K \varphi^{a}-J^{a} \varphi^{a}-\frac{3 N}{2 \lambda_{0}}\left(\chi-\mu_{0}^{2}\right)^{2}\right)\right\},
\end{aligned}
$$

where

$$
K \equiv-D^{2}+\hat{\xi} R+\chi
$$

The classical field equation for the auxiliary field is

$$
\chi=\mu_{0}^{2}+\frac{\lambda_{0}}{6 N} \varphi^{a} \varphi^{a},
$$

so that $\chi$ is related to the bound state we are looking for. Performing the trivial integration over $\varphi^{a}$, we obtain

$$
\begin{aligned}
\exp \left\{W\left[J^{a}\right]\right\} & =\int D \chi \exp \left\{W\left[J^{a}, \chi\right]\right\} \\
W\left[J^{a}, \chi\right]= & \int d^{4} x \sqrt{g}\left\{\frac{1}{2} J^{a} K^{-1} J^{a}+\frac{3 N}{2 \lambda_{0}}\left(\chi-\mu_{0}^{2}\right)^{2}\right\} \\
& -\frac{1}{2} N \operatorname{Tr} \ln [K] .
\end{aligned}
$$

All terms on the right-hand side (RHS) of Eq. (5) are of order $N$. Therefore, for $N$ very large, the $\chi$ integration can be evaluated by the saddle-point method. In leading order one has $W[J]=W[J, \bar{\chi}]+\cdots$, where $\bar{\chi}$ is the stationary point $(\delta W / \delta \chi)[J, \bar{\chi}]=0$. The corresponding effective action is

$$
\begin{aligned}
\Gamma\left[\phi^{a}, \chi\right]= & \int d^{4} x \sqrt{g}\left\{\frac{1}{2} \phi^{a}\left[-D^{2}+\hat{\xi}_{R}+\chi\right] \phi^{a}\right. \\
& \left.-\frac{3 N}{2 \lambda_{0}}\left(\chi-\mu_{0}^{2}\right)^{2}\right\} \\
& +\frac{1}{2} N \operatorname{Tr} \ln \left[-D^{2}+\hat{\xi} R+\chi\right],
\end{aligned}
$$

with $\bar{\chi}$ to be determined from $(\delta \Gamma / \delta \chi)[\phi, \bar{\chi}]=0$, or, explicitly,

$\bar{\chi}(x)=\mu_{0}^{2}+\frac{\lambda_{0}}{6 N} \phi^{2}(x)+\frac{\lambda_{0}}{6}\left\langle x\left|\left(-D^{2}+\hat{\xi}_{R}+\bar{\chi}\right)^{-1}\right| x\right\rangle$.

Similarly, the equation of motion $(\delta \Gamma / \delta \phi)[\bar{\phi}, \bar{\chi}]=0$ determines the vacuum expectation value $\bar{\phi} \equiv\langle\phi\rangle$. Because we are interested in the propagator of the $\varphi^{a} \varphi^{a}$ bound state, we shall expand the effective action $\Gamma$ around the vacuum configuration $(\bar{\phi}, \bar{\chi})$ allowing for small fluctuations of $\chi: \chi=\bar{\chi}+\delta \chi$. Isolating the piece quadratic in $\delta \chi$, we can read off the inverse propagator of $\chi$, including the induced $\xi$ term. Before we come to that, let us briefly recall the situation in flat space [5]. There, with the further assumption that $\phi^{2}$ is constant, Eq. (7) becomes the gap equation

$$
\bar{\chi}=\mu_{0}^{2}+\frac{\lambda_{0}}{6 N} \phi^{2}+\frac{\lambda_{0}}{6} \int \frac{d^{4} p}{(2 \pi)^{4}} \frac{1}{p^{2}+\bar{\chi}},
$$

and Eq. (6) gives rise to the effective potential $V_{\text {eff }}\left(\phi^{2}\right)$ related to $\bar{\chi}$ by

$$
\frac{\partial V_{\mathrm{eff}}\left(\phi^{2}\right)}{\partial \phi^{2}}=\frac{1}{2} \bar{\chi}\left(\phi^{2}\right) .
$$

The renormalized version of the gap equation (8) and the effective potential $V_{\text {eff }}$ have been extensively studied in Ref. [10]. In particular, it has been shown that there is no spontaneous symmetry breaking in the leading order of $1 / N$; i.e., the minimum of the effective potential always occurs at $\phi^{2}=0$. In view of Eq. (6) this means that

$$
\bar{\chi}\left(\phi^{2}=0\right) \equiv m^{2}
$$

is the mass square of the $\phi^{a}$ excitation.

In the following we assume that the gravitational field is sufficiently weak so that it has no significant influence on the internal structure of the composite particle and on the masses of $\phi^{a}$ and of the bound state, respectively [15]. Then we can use the same gap equation (8) as in flat space and work with a constant $\bar{\chi} \equiv m^{2}$. If we put $\phi^{a}=0$ and $\chi=m^{2}+\delta \chi$, Eq. (6) gives rise to a quadratic action of the general form

$$
\Gamma^{(2)}=\frac{1}{2} \int d^{4} x \sqrt{g} \delta \chi\left[\Pi\left(-D^{2}\right)+\xi_{0} R\right] \delta \chi,
$$

where $\Pi\left(-D^{2}\right)$ is the covariantized inverse $\chi$ propagator of flat space. Because the gravitational field is weak, the condition for $\delta \chi$ describing a propagating bound state of mass $m_{B}$ is given by $\Pi\left(p^{2}=-m_{B}^{2}\right)=0$, as in flat space. If the parameters $\lambda$ and $\mu^{2}$ are chosen appropriately, one finds that indeed $\Pi\left(p^{2}\right)$ can have a zero at negative Euclidean $p^{2}$. In the neighborhood of $p^{2}=-m_{B}^{2}$ we may expand $\Pi\left(p^{2}\right)$ :

$$
\Gamma^{(2)}=\frac{1}{2} \int d^{4} x \sqrt{g} \delta \chi\left[Z_{B}\left(-D^{2}+m_{B}^{2}\right)+\xi_{0} R\right] \delta \chi+\cdots,
$$

with

$$
Z_{B} \equiv \frac{\partial \Pi}{\partial p^{2}}\left(p^{2}=-m_{B}^{2}\right) .
$$

In order to obtain a conventionally normalized kinetic term, we introduce the renormalized field $B=Z_{B}^{1 / 2} \delta \chi$ and the renormalized $\xi$ parameter

$$
\xi=Z_{B}^{-1} \xi_{0},
$$

so that finally ${ }^{2}$

$$
\Gamma^{(2)}[B]=\frac{1}{2} \int d^{4} x \sqrt{g} B\left[-D^{2}+m_{B}^{2}+\xi R\right] B+\cdots .
$$

\footnotetext{
${ }^{2}$ Note that $\delta \chi, B$, and $Z_{B}$ have mass dimensions $+2,+1$, and -2 , respectively. As a consequence, the bare parameter $\xi_{0}$ has dimension -2 , but the renormalized $\xi$ is dimensionless, as it should be.
} 
Our aim is the determination of the renormalized parameter (14). Apart from $\xi_{0}$, we have to know the residue $Z_{B}$, which can be extracted from the calculation in flat space, however.

For a detailed discussion of the gap equation, which yields $\bar{\chi}$ as a function of $\mu^{2}$ and $\lambda$, and of the conditions for the occurrence of a stable bound state, we refer the reader to the work of Abbott, Kang, and Schnitzer [10]. Their results directly carry over to the present case, but we shall not repeat them here. Suffice it to say that the renormalized gap equation has two branches of solutions. On "branch I" the resulting bound states are tachyons, but on "branch II" stable $\left(Z_{B}>0\right)$ composite particles are possible. Their mass ranges from $m_{B}=0$ to $2 m$ depending on $\mu^{2}$ and $\lambda$. (For finite cutoff and positive bare coupling, only the tachyonic branch exists.)

Next we turn to the actual derivation of Eq. (11) which requires a partial evaluation of the functional trace on the RHS of Eq. (6). Using the proper time representation

$$
\begin{aligned}
\operatorname{Tr} \ln [- & \left.D^{2}+m^{2}+\hat{\xi} R+\delta \chi\right] \\
& =-\int_{0}^{\infty} \frac{d t}{t} e^{-m^{2} t} \operatorname{Tr} \exp \left\{-t\left(-D^{2}+\hat{\xi} R+\delta \chi\right)\right\},
\end{aligned}
$$

the relevant terms of the heat kernel $\operatorname{Tr} \exp (\cdots)$ can be found in the literature. Using the results of Barvinsky and Vilkovisky [16], the $\Pi$ term can also be found in this way:

$$
\begin{array}{r}
\operatorname{Tr} \exp \left\{-t\left(-D^{2}+\hat{\xi} R+\delta \chi\right)\right\} \\
=(4 \pi t)^{-2} \int d^{4} x \sqrt{g}\left\{1+\frac{1}{2} t^{2} \delta \chi \int_{0}^{1} d \alpha e^{\alpha(1-\alpha) t D^{2}} \delta \chi\right. \\
\left.+\frac{1}{2} t^{3} \delta \chi^{2} R\left(\frac{1}{6}-\hat{\xi}\right)+\cdots\right\} .
\end{array}
$$

Only terms of the form $\delta \chi D^{2 n} \delta \chi$ and $R \delta \chi^{2}$ were retained in this expansion. Inserting (17) into (6) with (16) and comparing to (11), one finds immediately,

$$
\begin{aligned}
\xi_{0} & =\frac{6 \hat{\xi}-1}{192 \pi^{2}} N \int_{0}^{\infty} d t e^{-m^{2} t} \\
& =\frac{6 \hat{\xi}-1}{192 \pi^{2}} \frac{N}{m^{2}},
\end{aligned}
$$

and, similarly,

$$
\Pi\left(-D^{2}\right)=-3 N \lambda_{0}^{-1}+3 N \bar{B}\left(-D^{2}\right),
$$

with

$$
\begin{aligned}
\bar{B}\left(p^{2}\right) \equiv-\frac{1}{96 \pi^{2}} \int_{0}^{1} d \alpha & \\
& \times \int_{0}^{\infty} \frac{d t}{t} \exp \left\{-t\left[m^{2}+\alpha(1-\alpha) p^{2}\right]\right\} .
\end{aligned}
$$

The $t$ integration on the RHS of Eq. (20) is logarithmically divergent for $t \rightarrow 0$. Keeping a finite cutoff $t_{0} \sim \Lambda^{-2}$, it is easy to show that (20) coincides with the usual loop integral (we use the notation of [10]):

$$
\begin{aligned}
\bar{B}\left(m^{2}, p^{2}, \Lambda^{2}\right) & =-\frac{1}{6} \int^{\Lambda} \frac{d^{4} q}{(2 \pi)^{4}} \frac{1}{\left[(p+q)^{2}+m^{2}\right]\left[q^{2}+m^{2}\right]} \\
& =-\frac{1}{96 \pi^{2}}\left[1+\ln \frac{\Lambda^{2}}{m^{2}}\right]+f\left(p^{2}, m^{2}\right)
\end{aligned}
$$

with

$$
\begin{aligned}
f\left(p^{2}, m^{2}\right)= & \frac{1}{48 \pi^{2}}\left[\frac{p^{2}+4 m^{2}}{p^{2}}\right]^{1 / 2} \\
& \times \ln \left[\frac{\left(p^{2}+4 m^{2}\right)^{1 / 2}+\sqrt{p^{2}}}{2 m}\right] .
\end{aligned}
$$

Equation (19) shows that the logarithmic divergence in (21) can be absorbed by a renormalization of $\lambda_{0}$. Combining Eqs. (13), (19), and (21), one obtains the finite result

$$
Z_{B}=3 N \frac{d f}{d p^{2}}\left(p^{2}=-m_{B}^{2}, m^{2}\right) .
$$

The analytic continuation of (22) to negative values of $p^{2}$ yields the desired answer (valid for $m_{B}<2 m$ ):

$$
\begin{aligned}
Z_{B}=\frac{N}{128 \pi^{2} m^{2} v^{3}}\left[\left(1-v^{2}\right)^{-1 / 2} \arcsin (v)-v\right], & \\
v & \equiv \frac{m_{B}}{2 m} .
\end{aligned}
$$

Together with the expression (18) for $\xi_{0}$, this leads to the renormalized $\xi$ parameter

$\xi(v)=\frac{2}{3}(6 \hat{\xi}-1) v^{3}\left[\left(1-v^{2}\right)^{-1 / 2} \arcsin (v)-v\right]^{-1}$.

Equation (25) is our final result. The value of $\xi$ depends only on the ratio $v=m_{B} / 2 m \in[0,1]$, where $m_{B}$ and $m$ are the masses of the bound state and the elementary $\phi^{a}$ particle, respectively, as well as on $\hat{\xi}$, the $\xi$ parameter of $\phi$ itself. If $\phi$ is minimally coupled, $\xi=0, \xi(v)$ can assume values in the interval $[-1,0]$ only. In particular, the "conformal" value $\xi=\frac{1}{6}$ cannot be realized. If, on the other hand, the $\phi$ field is coupled with $\hat{\xi}=\frac{1}{6}$, then $\xi(v)=0$ for all $v$ so that the bound state is minimally coupled. In the strong binding limit, when $m_{B}$ is (almost) zero, ${ }^{3} \mathrm{Eq}$. (25) reduces to $\xi(0)=6 \hat{\xi}-1$, i.e., for $\hat{\xi}=0$ the lower bound $\xi=-1$ is assumed. From the analysis in Ref. [10] we know that the mass of a stable bound state cannot exceed $2 m$. In the limit $m_{B} \rightarrow 2 m$, the $\xi$ parameter vanishes as

$$
\xi\left(m_{B} \rightarrow 2 m\right)=\frac{2}{3}(6 \hat{\xi}-1) \frac{1}{\pi m}\left[4 m^{2}-m_{B}^{2}\right]^{1 / 2} .
$$

At the same time the wave-function renormalization $Z_{B}$ of Eq. (24) diverges, indicating that $m_{B}$ cannot be pushed

\footnotetext{
${ }^{3}$ Inspired by recent work on matrix models a double scaling limit leading to a critical theory with $m_{B}=0$ has been suggested [17]. In four dimensions its existence has been questioned [18], however, because precisely at the critical point the effective potential becomes everywhere complex.
} 
beyond $2 m$. As $v$ is increased from $v=0$ to $1, \xi$ starts at $6 \hat{\xi}-1$ and monotonically approaches zero.

\section{ADDING EXPLICIT SYMMETRY BREAKING}

It has been pointed out [13] that if both spontaneous and explicit chiral symmetry breaking occur in models with a large number of Goldstone bosons, then a stable scalar resonance might form which can be regarded as a bound state of "pions." Chivukula and Golden [14] (CG) have investigated this possibility in the context of the $\mathrm{O}(N)$ linear $\sigma$ model. They find that for weak coupling there are indeed stable bound states which become broad resonances when the coupling becomes strong. In this section we study their nonminimal coupling to gravity.

CG add for $\mu_{0}^{2}<0$ an explicit symmetry-breaking term

$$
S_{\mathrm{SB}}=-\alpha \int d^{4} x \sqrt{g} \varphi_{N}
$$

to the action (2). This forces the vacuum expectation value $\left\langle\varphi^{a}\right\rangle$ to lie along the $\varphi_{N}$ direction. The fields $\varphi_{1}, \ldots, \varphi_{N-1}$ correspond to the pions. Because of the explicit symmetry breaking, they become massive, but the unbroken $\mathrm{O}(N-1)$ symmetry guarantees that they are degenerate in mass. The inclusion of the term (27) leads to the effective action

$$
\Gamma_{\alpha}\left[\phi^{a}, \chi\right]=\Gamma\left[\phi^{a}, \chi\right]-\alpha \int d^{4} x \sqrt{g} \phi^{N},
$$

where $\Gamma\left[\phi^{a}, \chi\right]$ is the previous expression (6). Differentiating (28) with respect to $\chi$ leads to the gap equation (7) again. The condition $\left(\delta \Gamma_{\alpha} / \delta \phi^{a}\right)[\langle\phi\rangle, \bar{\chi}]=0$ gives rise to

$$
\begin{aligned}
& {\left[-D^{2}+\hat{\xi} R+\bar{\chi}\right]\left\langle\phi^{j}\right\rangle=0, j=1, \ldots, N-1} \\
& {\left[-D^{2}+\hat{\xi} R+\bar{\chi}\right]\left\langle\phi^{N}\right\rangle=\alpha .}
\end{aligned}
$$

Again we assume that $R$ is constant so that $\left\langle\phi^{a}\right\rangle$ and $\bar{\chi}$ become $x$ independent too. Then (29) implies

$$
\left\langle\phi^{j}\right\rangle=0,\left\langle\phi^{N}\right\rangle=\alpha[\bar{\chi}+\hat{\xi} R]^{-1} .
$$

Next we expand $\Gamma_{\alpha}\left[\phi^{a}, \chi\right]$ about its minimum. Writing

$$
\begin{aligned}
& \phi^{j} \equiv \pi_{j}, \quad j=1, \ldots, N-1, \\
& \phi^{N} \equiv\left\langle\phi^{N}\right\rangle+\sigma(x), \\
& \chi \equiv \bar{\chi}+\tau(x) \equiv m_{\pi}^{2}+\tau(x)
\end{aligned}
$$

we obtain

$$
\begin{aligned}
\Gamma\left[\pi_{j}, \sigma, \tau\right]=\int d^{4} x \sqrt{g}\left\{\frac{1}{2} \pi_{j}\left[-D^{2}+\hat{\xi} R+m_{\pi}^{2}+\tau\right] \pi_{j}+\frac{1}{2} \sigma\left[-D^{2}+\hat{\xi} R+m_{\pi}^{2}+\tau\right] \sigma+\left\langle\phi^{N}\right\rangle \tau \sigma-\frac{3 N}{2 \lambda_{0}} \tau^{2}\right. \\
\left.+\tau\left[\frac{3 N \mu_{0}^{2}}{\lambda_{0}}-\frac{3 N}{\lambda_{0}} m_{\pi}^{2}+\frac{1}{2}\left\langle\phi^{N}\right\rangle^{2}\right]\right\}+\frac{N}{2} \operatorname{Tr} \ln \left[-D^{2}+\hat{\xi} R+m_{\pi}^{2}+\tau\right]
\end{aligned}
$$

We see that $\bar{\chi} \equiv m_{\pi}^{2}$ is indeed the mass square of the pions. Their nonminimal coupling to gravity is given by the classical value $\hat{\xi}$. Let us now analyze the propagator of the coupled $\sigma-\tau$ system. From our previous calculation we know already that

$$
\begin{aligned}
& \frac{N}{2} \operatorname{Tr} \ln \left[-D^{2}+\hat{\xi} R+m_{\pi}^{2}+\tau\right] \\
& =\frac{1}{2} \int d^{4} x \sqrt{g} \tau(x)\left[3 N \bar{B}\left(m_{\pi}^{2},-D^{2}, \Lambda^{2}\right)+\xi_{0} R\right] \tau(x) \\
& \quad+O\left(\tau^{3}\right)
\end{aligned}
$$

where $\xi_{0}$ is given in (18). Hence the quadratic action for $\sigma$ and $\tau$ is

$$
\Gamma_{\sigma \tau}^{(2)}=\frac{1}{2} \int d^{4} x \sqrt{g}(\sigma, \tau) K\left(-D^{2}, R\right)(\sigma, \tau)^{T},
$$

with the inverse propagator

$$
\begin{aligned}
& K_{\sigma \sigma}=-D^{2}+\hat{\xi} R+m_{\pi}^{2}, \\
& K_{\sigma \tau}=K_{\tau \sigma}=\left\langle\phi^{N}\right\rangle, \\
& K_{\tau \tau}=-3 N \lambda_{0}^{-1}+3 N \bar{B}\left(m_{\pi}^{2},-D^{2}, \Lambda^{2}\right)+\xi_{0} R .
\end{aligned}
$$

Let us define a renormalized, scale-dependent coupling $\lambda(M)$ by absorbing the divergent part of $\bar{B}$ into $\lambda_{0}^{-1}$. We set [10]

$\lambda_{0}^{-1}-\bar{B}\left(m_{\pi}^{2}, p^{2}, \Lambda^{2}\right)=\lambda(M)^{-1}-B\left(m_{\pi}^{2}, p^{2}, M^{2}\right)$, where

$$
\begin{aligned}
B\left(m_{\pi}^{2}, p^{2}, M^{2}\right) & \equiv \bar{B}\left(m_{\pi}^{2}, p^{2}, \Lambda^{2}\right)-\bar{B}\left(M^{2}, 0, \Lambda^{2}\right) \\
& =\frac{1}{96 \pi^{2}} \ln \frac{m_{\pi}^{2}}{M^{2}}+f\left(p^{2}, m_{\pi}^{2}\right)-f\left(0, M^{2}\right)
\end{aligned}
$$

is finite for $\Lambda \rightarrow \infty$. The coupling $\lambda(M)$ shows the usual (one-loop) running: it increases monotonically (logarithmically, in fact) with $M$ and finally becomes infinite at a certain scale ("Landau pole"). We use the convention of CG and set the scale $M$ equal to the Landau scale so that $1 / \lambda(M)=0$. Instead of the renormalized coupling, we use the magnitude of $M$ (relative to $m_{\pi}$ or $\left\langle\phi^{N}\right\rangle$, say) to characterize the strength of the quartic interaction. Let us assume we specify the bare parameters of the theory at a UV cutoff scale $\Lambda$ which is slightly below $M$. Then we may start from a large, positive $\lambda_{0}$ and obtain a smaller renormalized $\lambda$ by following the renormalization-group flow from high to low scales. Clearly when $M$, and hence $\Lambda$, are large (small), we have to evolve $\lambda$ for a long (short) "renormalization-group time" and therefore the renormalized coupling is small (large). Hence in this parametrization the weak-coupling regime obtains, for $M \gg\left\langle\phi^{N}\right\rangle, m_{\pi}$.

The inverse propagator reads now

$K\left(-D^{2}, R\right)$ 


$$
=\left[\begin{array}{cc}
-D^{2}+\hat{\xi}_{R}+m_{\pi}^{2} & \left\langle\phi^{N}\right\rangle \\
\left\langle\phi^{N}\right\rangle & 3 N B\left(m_{\pi}^{2},-D^{2}, M^{2}\right)+\xi_{0} R
\end{array}\right] .
$$

In order to decouple the modes, we have to bring it to the diagonal form

$$
K\left(-D^{2}, R\right)=\left[\begin{array}{cc}
e_{+}\left(-D^{2}, R\right) & 0 \\
0 & e_{-}\left(-D^{2}, R\right)
\end{array}\right] .
$$

If a bound state occurs for some $p^{2}=-m_{B}^{2}<0$, only one of the eigenvalues $\left(e_{-}\right.$, say) will have a zero. Near the zero, we expect a behavior of the form

$e_{-}\left(-D^{2}, R\right)=Z_{(-)}\left(-D^{2}+m_{B}^{2}\right)+\xi_{(-)} R+\cdots$

so that the renormalized $\xi$ parameter of the bound state is given by

$$
\xi=Z_{(-)}^{-1} \xi_{(-)} \text {. }
$$

For our purposes it is sufficient to determine the location of the zero, $m_{B}^{2}$, and its residue $Z_{(-)}$in the absence of gravity because we have to keep only terms of lowest order in the curvature. Setting $s \equiv-p^{2}>0$, the eigenvalues of $K(-s, 0)$ are, to leading order in $1 / N$,

$$
\begin{aligned}
e_{ \pm}(s)= & \frac{1}{2}\left[m_{\pi}^{2}-s+3 N B\right] \\
& \times\left\{1 \pm\left[1-\frac{4 G(s)}{\left(m_{\pi}^{2}-s+3 N B\right)^{2}}\right]^{1 / 2}\right\},
\end{aligned}
$$

where $B \equiv B\left(m_{\pi}^{2},-s, M^{2}\right)$ and

$$
\begin{aligned}
G(s) & \equiv \operatorname{det} K(-s, 0) \\
& =3 N\left(m_{\pi}^{2}-s\right) B\left(m_{\pi}^{2},-s, M^{2}\right)-\left\langle\phi^{N}\right\rangle^{2} .
\end{aligned}
$$

If there exists a value $s_{0} \equiv m_{B}^{2}>0$ for which $G\left(s_{0}\right)=0$, then the eigenvalue $e_{-}$vanishes and the corresponding eigenmode represents a bound state of the pions with mass $m_{B}$. Expanding $e_{-}(s)$ near $s=s_{0}$ and comparing to (40), we obtain, for the residue,

$$
Z_{(-)}=\left.\frac{s_{0}-m_{\pi}^{2}}{f^{2}} \frac{d G}{d s}\right|_{s=s_{0}}+O\left(\frac{1}{N}\right) .
$$

Here we used the notation $\left\langle\phi^{N}\right\rangle \equiv f$ because it can be shown [14] that the vacuum expectation value of $\phi^{N}$ equals the pion decay constant in this model. Employing Eq. (37), we find explicitly

$$
\begin{aligned}
\frac{d G}{d s}= & \frac{N}{16 \pi^{2}}\left[1+\ln \left[\frac{M}{m_{\pi}}\right]+\frac{m_{\pi}^{2}-s}{2 s}\right] \\
& -3 N\left[1+\frac{2 m_{\pi}^{2}}{s} \frac{m_{\pi}^{2}-s}{4 m_{\pi}^{2}-s}\right] \tilde{f}\left(s, m_{\pi}^{2}\right),
\end{aligned}
$$

where $\widetilde{f}\left(s, m_{\pi}^{2}\right) \equiv f\left(-s, m_{\pi}^{2}\right)$ is the analytic continuation of (22) to negative values of $p^{2}=-s, 0<s<4 m_{\pi}^{2}$ :

$\widetilde{f}\left(s, m_{\pi}^{2}\right)=\frac{1}{48 \pi^{2}}\left[\frac{4 m_{\pi}^{2}-s}{s}\right]^{1 / 2} \arctan \left(\frac{s}{4 m_{\pi}^{2}-s}\right)^{1 / 2}$.
Finally, we have to determine $\xi_{(-)}$, in the expansion (40). It is sufficient to diagonalize $K\left(-D^{2}, R\right)$ to first order in the (constant) curvature $R$. Omitting terms which are of higher order in $1 / N$, we find, after some algebra,

$$
\xi_{(-)}=\hat{\xi}+\frac{4}{3} \pi^{2}(1-6 \hat{\xi}) \frac{f^{2} / N}{m_{\pi}^{2}}\left[\ln \left(\frac{M}{m_{\pi}}\right)\right]^{-2}+O\left(\frac{1}{N}\right) \text {. }
$$

If we divide by $Z_{(-)}$, we arrive at the following result for the renormalized parameter:

$$
\begin{aligned}
\xi= & \frac{f^{2}}{\left(s_{0}-m_{\pi}^{2}\right) G^{\prime}\left(s_{0}\right)} \\
& \times\left\{\hat{\xi}-\frac{4}{3} \pi^{2}(1-6 \hat{\xi}) \frac{f^{2} / N}{m_{\pi}^{2}}\left[\ln \left[\frac{M}{m_{\pi}}\right]\right]^{-2}\right\} .
\end{aligned}
$$

Here, $G^{\prime}=d G / d s$ is given by Eq. (45) with (46). Our result is of order $N^{0}$ because both $G^{\prime}$ and $f^{2}[14]$ are of order $N$.

The essential features of the result are as follows. Excluding wave-function effects, the induced $\xi$ parameter is given by the expression (47) which has no explicit dependence on $s_{0}$. It consists of a classical piece $\hat{\xi}$ and a quantum correction which is proportional to $(\ln M)^{-2}$. It vanishes in the weak-coupling regime where $M$ is large. Furthermore, it is identically zero if $\hat{\xi}$ assumes the "conformal" value $\hat{\xi}=\frac{1}{6}$. Including the wave-function renormalization, Eq. (48), the expression for $\xi$ contains the (mass) ${ }^{2}$ of the bound state $s_{0}$ explicitly. It is a complicated function of the parameters specifying the model: the scale $M$, the pion mass $m_{\pi}$, and the pion decay constant $f$. In Ref. [14] the dependence of the dimensionless ratio $s_{0} / f^{2}$ on $M / f$ and $m_{\pi} / f$ has been determined by solving numerically the equation $G\left(s_{0}\right)=0$ in the complex $s$ plane. It turns out that if the theory is weakly coupled ( $M$ large), this equation can be satisfied by a real $s_{0}$ in the interval $\left[0,4 m_{\pi}^{2}\right]$. This is the bound state we are interested in. There exists also a tachyonic zero, with a much higher mass $m_{t}$, however. The theory is meaningful as an effective theory at scales well below $m_{t}$. When the coupling is increased ( $M$ is decreased), the bound-state pole moves on the positive real axis of the complex $s$ plane to the left, and finally collides with another pole which comes from smaller values of $s$ and moves to the right. After the collision, the two poles move off the real axis, with complex-conjugate residues whose real part is positive. Thus the stable bound state turns into an increasingly unstable resonance. For any $s_{0}=s_{0}\left(M, m_{\pi}, f\right)$, Eq. (48) gives the $\xi$ parameter of the corresponding scalarisoscalar excitation, at least as long as $s_{0}$ remains real and there is a stable bound state. If $s_{0}$ is complex but has an imaginary part much smaller than its real part, then the composite particle is almost stable and $\xi$ has its usual interpretation.

In the weak binding limit, when $s_{0}$ is not too far below the threshold $4 m_{\pi}^{2}$, Eq. (48) can be approximated by 


$$
\begin{aligned}
\xi= & \frac{256 \pi}{9} \frac{f^{2} / N}{m_{\pi}^{2}}\left[1-\frac{s_{0}}{4 m_{\pi}^{2}}\right]^{1 / 2} \\
& \times\left\{\hat{\xi}-\frac{4 \pi^{2}}{3}(1-6 \hat{\xi}) \frac{f^{2} / N}{m_{\pi}^{2}}\left[\ln \left[\frac{M}{m_{\pi}}\right]\right]^{-2}\right\} .
\end{aligned}
$$

Obviously, $\xi$ vanishes if $s_{0}$ approaches the threshold $4 \mathrm{~m}_{\pi}^{2}$ from below. At this point, $Z_{(-)}$diverges and there is no bound state anymore.

\section{CONCLUSIONS}

In this paper we have investigated two models with fundamental scalar fields for which the existence of bound states can be established in the large- $N$ limit. We have shown how in both models the $\xi R \phi^{2}$-type nonminimal coupling of the bound states to gravity can be calculated unambiguously in terms of the renormalized parameters characterizing the theory.

In the $\mathrm{O}(N)$-symmetric vector model we found that the induced $\xi$ parameter of the bound state depends only on the mass ratio $m_{B} / m$ and on the nonminimal coupling of the fundamental field. If the latter is minimally coupled, $\xi$ is found to range between -1 and 0 . This has to be compared to the "conformal" value $\xi=\frac{1}{6}$ which was found [1] for the bound state of the Nambu-Jona-Lasinio model. As mentioned in the Introduction, a negative value of $\xi$ would be rather attractive from the point of view of inflationary cosmology. The two models are different in that the $\mathrm{O}(N)$-symmetric scalar model allows for a nonminimal dimension-4 coupling $(\hat{\xi})$ already at the level of the constituent particles. We have seen that for any allowed value of $m_{B} / m$, the $\xi$ parameter of the bound state lies between $6 \hat{\xi}-1$ and zero. It is remarkable that it is precisely when the fundamental scalars have the conformally invariant coupling $\xi=\frac{1}{6}$ that the bound states turn out to be minimally coupled. We also studied a model in which the $\mathrm{O}(N)$ symmetry is broken both spontaneously and explicitly. Because of the symmetrybreaking term, the Goldstone bosons acquire a mass. Under certain conditions these "pions" can form stable bound states [14]. This requires that the theory be weakly coupled. For strong coupling the bound states become increasingly broad resonances. Contrary to our first model, the second one is meaningful also as an effectivefield theory with a finite ultraviolet cutoff. For the bound states of the $\mathrm{O}(N)$-symmetric vector model to exist, it is important that we work with the renormalized form of the theory with the cutoff sent to infinity. This means that the bare coupling becomes large and negative finally [19]. On the other hand, in the model with symmetry breaking from $\mathrm{O}(N)$ to $\mathrm{O}(N-1)$, interesting effects occur even for small couplings which might be forced upon us by the triviality bounds. As for the nonminimal gravitational coupling, an essential difference between the two models is best seen by looking at the induced $\xi$ parameter with the wave-function renormalization not yet included. In Eq. (47) we obtained for the second model a result of the form $\xi=\hat{\xi}+$ (quantum corrections). We observe that in this case the actual $\xi$ parameter is basically given by the $\hat{\xi}$ parameter of the constituents which is only slightly renormalized by quantum effects. The situation is different in the first, strongly coupled model. From Eq. (18) and its derivation, it is clear that the $\xi_{0}$ which obtains there, though depending on $\hat{\xi}$, is a pure quantum effect; i.e., it is not of the form $\hat{\xi}+$ (small corrections). The lesson to be learned here is that for weakly coupled theories it is even more important to know the detailed properties of the constituents in order to predict the $\xi$ parameter of their bound states.
[1] C. T. Hill and D. S. Salopek, Ann. Phys. (N.Y.) 213, 21 (1992).

[2] Y. Nambu and G. Jona-Lasinio, Phys. Rev. 122, 345 (1961).

[3] L. Parker and D. J. Toms, Phys. Rev. D 29, 1584 (1984).

[4] I. M. Lichtzier and S. D. Odintsov, Europhys. Lett. 7, 95 (1988).

[5] T. Muta and S. D. Odintsov, Mod. Phys. Lett. A 6, 3641 (1991).

[6] B. L. Spokoiny, Phys. Lett. 129B, 39 (1984); R. Fakir and W. G. Unruh, Phys. Rev. D 41, 1783 (1990); D. S. Salopek, J. R. Bond, and J. M. Bardeen, ibid. 40, 1753 (1989).

[7] D. La and P. J. Steinhardt, Phys. Rev. Lett. 62, 376 (1989).

[8] H. J. Schnitzer, Phys. Rev. D 10, 1800 (1974); see also J. Zinn-Justin, Quantum Field Theory and Critical Phenomena (Oxford University Press, Oxford, England, 1989); R. J. Rivers, Path Integral Methods in Quantum Field Theory (Cambridge University Press, Cambridge, England, 1988).

[9] S. Coleman, R. Jackiw, and H. D. Politzer, Phys. Rev. D 10, 2491 (1974).

[10] L. F. Abbott, J. S. Kang, and H. J. Schnitzer, Phys. Rev. D 13, 2212 (1976).

[11] W. A. Bardeen and M. Moshe, Phys. Rev. D 28, 1372 (1983).
[12] M. Reuter, N. Tetradis, and C. Wetterich, Nucl. Phys. B401, 567 (1993).

[13] R. N. Cahn and M. Suzuki, Phys. Rev. Lett. 67, 169 (1991).

[14] R. S. Chivukula and M. Golden, Nucl. Phys. B372, 44 (1992).

[15] For a discussion of curvature-induced $\mathrm{O}(N)$-symmetry breaking, see, for instance, P. R. Anderson and R. Holman, Phys. Rev. D 34, 2277 (1986); S. D. Odintsov, Europhys. Lett. 7, 1 (1988). A similar analysis of the Nambu-Jona-Lasinio model is contained in T. Inagaki et al., Mod. Phys. Lett. A 8, 2117 (1993).

[16] A. O. Barvinsky and G. A. Vilkovisky, Nucl. Phys. B282, 163 (1987); B333, 471 (1990); B333, 512 (1990); Report No. CERN-TH 6392/92, 1992 (unpublished).

[17] J. Zinn-Justin, Phys. Lett. B 257, 335 (1991); P. Di Vecchia, M. Kato, and N. Ohta, Int. J. Mod. Phys. A 7, 1391 (1992).

[18] H. J. Schnitzer, Mod. Phys. Lett. A 7, 2449 (1992); for the situation for $d=2$, see P. Di Vecchia and M. Moshe, Report No. Technion-PH-54-92, 1992 (unpublished).

[19] For a related discussion, see also J. Nunes and H. J. Schnitzer, Report No. BRX-TH-351; hep-ph/9311319 (unpublished). 